\title{
Vector Modal Solution of Evanescent Coupler
}

\author{
Mehmet S. Dinleyici and David B. Patterson, Member, IEEE
}

\begin{abstract}
We present a vector modal solution for the evanescent coupler comprising an optical fiber and a slab waveguide. We identify the normal vector ridge modes of the device for different configurations. The dispersion characteristics and the power transmission properties of these modes are presented. Also, the effect of the proximity between the waveguides on the ridge modes is investigated.
\end{abstract}

Index Terms-Dielectric waveguides, optical waveguide components, waveguide couplers.

\section{INTRODUCTION}

$\mathbf{T}$ HE fiber-to-planar waveguide coupler system has been studied recently by a number of researchers [1]-[3] because of the wide variety of applications for this in-line component in fiber optic communication systems. Some of these applications are wavelength division multiplexing (WDM), in-line fiber modulators and tunable filters [4]-[7]. A mathematical model used by many researchers has been developed by Marcuse [3] using coupled mode theory (CMT). This model assumes only weak perturbation of the normal modes of the individual waveguides, namely, the fiber and the slab. However in the close proximity of the guides employed in these components (where the guides may be in contact), these modes are highly perturbed, and their propagation constants can be affected to the degree that CMT is no longer valid. As Marcuse notes in [3], the modes obtained by CMT are not sufficient to construct a mathematical model which will explain the observed behavior of these devices. Also, as Johnstone states in [4], the wavelength-dependent loss mechanism in the system is not fully understood.

In this paper we have adapted a mode expansion model, first introduced by Marcuse [8] for the modeling of Dfiber modes, to identify the approximate ridge modes of the fiber/slab system. We then use the characteristics of these modes to explain the experimentally observed device behaviors, including the dispersion relations of the system and the power transmission properties. It is important to clarify our definitions of modes here. Ridge modes are defined as hybrid modes of the fiber-slab system that are confined in both transverse directions, while unbounded modes are defined as slab-guided solutions that extend indefinitely in the direction along the slab interface. Thus, the isolated fiber mode is a ridge mode solution (if the core index exceeds the slab index),

Manuscript received June 2, 1997; revised August 18, 1997. The work of M. S. Dinleyici was supported by a scholarship provided by Izmir Institute of Technology, Turkey.

The authors are with the Electrical and Computer Engineering Department, Illinois Institute of Technology, Chicago, IL 60616 USA.

Publisher Item Identifier S 0733-8724(97)08870-1. but the isolated slab mode solution is unbounded, representing the cut-off condition of the ridge mode.

We first define the problem, providing the mode expansion functions and outlining the method of solution for the stated geometries. We also provide corrections to some errors that we have identified in the derivations of [8]. These errors comprise sign errors and transposed subscripts which lead to significant errors in the field calculations, particularly in the case of weak guidance (i.e., small normalized frequency, $V$ ) in the fiber. We note that, for large $V$ in the symmetric coupler cases considered in [8], the resulting errors are small and not likely to be recognized.

We next analyze the numerical solutions to the problem, varying the fiber-slab distance, the slab refractive index, and the fiber core refractive index. The solutions obtained are those having electrical field nearly perpendicular to the slab/cladding boundary, quasi-TM modes, and those having electrical field nearly parallel to this boundary, quasi-TE modes. For the weakly-guiding parameters assumed in this paper, the mode solutions are nearly degenerate quasi-TM and quasi-TE modes. However, the full vectorial analysis provided here is applicable to the general case with no assumption of weak guidance. We analyze the problem for $q$-TM modes, with the $q$-TE mode relations provided in Appendix C.

We show that two types of modes are present in the hybrid structure: confined ridge modes and unbounded continuous modes. We characterize the behavior of the ridge modes, and use these modes to model the behavior of the fiber/slab coupling system, providing theoretical curves for the expected transmission properties and tunability of such a device.

\section{Mathematical Model}

We begin by describing the optical modes of the fiber/slab structure with expansions into the orthonormal functions characteristic of the local geometries of the various regions. This approach, first developed by Marcuse [8] to describe the coupling between a D-fiber and a semi-infinite cladding, is adapted here to include a finite-width slab region between the fiber cladding and a semi-infinite region.

In Fig. 1, a schematic representation of the composite waveguide with relevant geometries and optical parameters is shown. In this geometry, the planar waveguide (PWG) is not bounded in the $y$-direction, and the circular fiber cladding is assumed sufficiently large so that no significant reflection occurs at the cladding-air boundary (e.g., at the large negative value of $x$ ). A core of uniform refractive index $n_{1}$ and radius $\rho$ is centered at the origin of the coordinate system, surrounded by a cladding of index $n_{2}$. The cladding/PWG interface, in the $y$ - $z$ plane, is located a distance $d$ from the core-cladding 


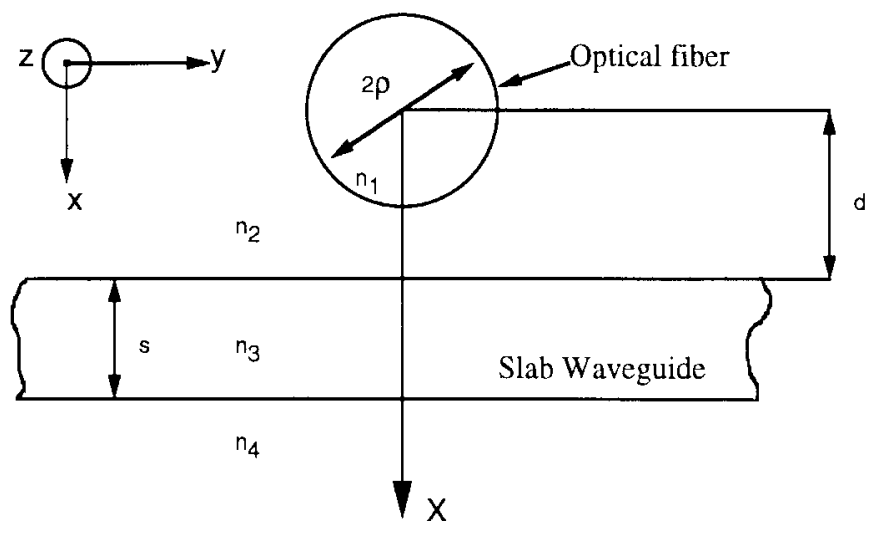

Fig. 1. Geometry of the evanescent coupler.

boundary in the positive $x$-direction. The PWG has thickness $s$ and refractive index $n_{3}$. Outside the slab region is a semiinfinite cover region with index $n_{4}$.

We begin by assuming monochromatic light, with a frequency dependence like $\exp \{-j \beta t\}$, and find the general solutions to the vector Helmholtz equation describing optical propagation in the various locally-homogeneous regions. Clearly, the geometries of the different regions lead to logical orthogonal function expansions in either Cartesian or cylindrical coordinate systems. These functions have the common feature of propagation in the positive $z$-direction with a dependence like $\exp \{j \omega z\}$.

Using the symmetry of the system, we may represent the $z$-components of the electric and magnetic fields in the core for $q$-TM modes as

$$
\begin{aligned}
& e_{z 1}=\sum_{n=0}^{N} A_{n}^{(1)} J_{n}(U R) \cos (n \phi) \\
& h_{z 1}=\sum_{n=0}^{N} A_{n}^{(2)} J_{n}(U R) \sin (n \phi)
\end{aligned}
$$

where $U=\rho\left(k^{2} n_{1}^{2}-\beta^{2}\right)^{1 / 2} ; R=r / \rho ; k=2 \pi / \lambda$ and $R$ is the normalized radius. Also, $A_{n}^{(1)}, A_{n}^{(2)}$ are the fields coefficients in the core. The expansion functions $J_{n}(U R)$ are Bessel functions of the first kind with order $n$. Note that $\phi=0$ corresponds to $y=0$ in Fig. 1. The cladding fields may be expanded in terms of modified Bessel functions $I_{n}$ and $K_{n}$

$$
\begin{aligned}
& e_{z 2}=\sum_{n=0}^{N}\left(B_{n}^{(1)} K_{n}(W R)+B_{n}^{(2)} I_{n}(W R)\right) \cos (n \phi) \\
& h_{z 2}=\sum_{n=0}^{N}\left(B_{n}^{(3)} K_{n}(W R)+B_{n}^{(4)} I_{n}(W R)\right) \sin (n \phi)
\end{aligned}
$$

where $W=\rho\left(\beta^{2}-k^{2} n_{2}^{2}\right)^{1 / 2}$. The terms $\sin (n \phi)$ and $\cos (n \phi)$ can be interchanged in (1), (2) to form a second set of solutions ( $q$-TE modes) with polarization orthogonal to those of (1). The expressions for $q$-TE modes are provided in Appendix C. Matching fields of the cladding and the core at the boundary $R=1$, we may express the coefficients $A_{n}$ as linear combinations of coefficients $B_{n}$.

The fields of the slab may be expressed as a superposition of plane waves having the lack of a favorable propagation direction in the $y-z$ plane, but bounded in the $x$-direction

$$
\begin{array}{r}
e_{z 3}=\int_{-\infty}^{\infty} \exp (j \nu Y)\left(C_{\nu}^{(1)} e^{-j \tau(X-D)}\right. \\
\left.+C_{\nu}^{(2)} e^{j \tau(X-D)}\right) d \nu \\
h_{z 3}=\int_{-\infty}^{\infty} \exp (j \nu Y)\left(C_{\nu}^{(3)} e^{-j \tau(X-D)}\right. \\
\left.+C_{\nu}^{(4)} e^{j \tau(X-D)}\right) d \nu
\end{array}
$$

where $\tau^{2}+\nu^{2}=\rho^{2}\left(k^{2} n_{3}^{2}-\beta^{2}\right)$ and $D$ is the normalized separation distance, $D=d / \rho$. All distances represented via capital letters are normalized to the core radius, $\rho$. The fields in the semi-infinite cover region outside the slab, assuming boundedness in the $x$-direction, have the form

$$
\begin{aligned}
& e_{z 4}=\int_{-\infty}^{\infty} D_{\nu}^{(1)} \exp (-\gamma(X-L)) \exp (j \nu Y) d \nu \\
& h_{z 4}=\int_{-\infty}^{\infty} D_{\nu}^{(2)} \exp (-\gamma(X-L)) \exp (j \nu Y) d \nu
\end{aligned}
$$

where $\nu^{2}-\gamma^{2}=\left(k^{2} n_{4}^{2}-\beta^{2}\right) \rho^{2}$ and $L$ is the normalized distance from the origin to the slab/cover medium boundary. That is, $L=(d+s) / \rho$. When the tangential fields of the slab and the cover medium are matched at $X=L$, four independent equations are obtained. By eliminating the coefficients $D_{\nu}^{(1)}$ and $D_{\nu}^{(2)}$ from these four equations, the following two sets of equations are found:

$$
\begin{aligned}
& C_{\nu}^{(3)} \exp (-2 \tau S) Z_{0} k\left(j Q_{3}^{2} \gamma+Q_{4}^{2} \tau\right) \\
&+C_{\nu}^{(4)}\left(j Q_{3}^{2} \gamma-Q_{4}^{2} \tau\right) Z_{0} k \\
&=\left(C_{\nu}^{(2)}+C_{\nu}^{(1)} \exp (-2 \tau S)\right) \beta V V_{3}^{2} \\
& C_{\nu}^{(2)} \frac{k}{Z_{0}}\left(n_{3}^{2} \tau Q_{4}^{2}-j n_{4}^{2} \gamma Q_{3}^{2}\right)-C_{\nu}^{(1)} \exp (-2 \tau S) \\
& \\
& \cdot \frac{k}{Z_{0}}\left(j n_{4}^{2} \gamma Q_{3}^{2}+Q_{4}^{2} n_{3}^{2} \tau\right) \\
&=\left(C_{\nu}^{(4)}+C_{\nu}^{(3)} \exp (-2 \tau S)\right) \beta V_{3}^{2}
\end{aligned}
$$

where the terms $Q_{2}, Q_{3}$, and $Q_{4}$ represent normalized transverse propagation constants in the various regions and are defined as $Q_{2}^{2}=\left(k^{2} n_{2}^{2}-\beta^{2}\right) \rho^{2}, Q_{3}^{2}=\left(k^{2} n_{3}^{2}-\beta^{2}\right) \rho^{2}$, and $Q_{4}^{2}=\left(k^{2} n_{4}^{2}-\beta^{2}\right) \rho^{2}$. The terms $V_{2}$ and $V_{3}$ are normalized frequencies of the slab $V_{2}^{2}=\rho^{2} k^{2}\left(n_{3}^{2}-n_{2}^{2}\right), V_{3}^{2}=$ $\rho^{2} k^{2}\left(n_{3}^{2}-n_{4}^{2}\right), V$ is the normalized frequency of the fiber $V^{2}=k^{2} \rho^{2}\left(n_{1}^{2}-n_{2}^{2}\right)$ and $Z_{0}$ is the free space impedance.

While the fields at the slab-cladding interface may be matched directly, we instead follow the approach of [1] in expressing the cladding fields in Cartesian coordinates:

$$
\begin{aligned}
& e_{z}=\int_{-\infty}^{\infty} \exp (j \nu y)\left(P_{\nu} e^{-\sigma(X-D)}+Q_{\nu} e^{\sigma(X-D)}\right) d \nu \\
& h_{z}=\int_{-\infty}^{\infty} \exp (j \nu y)\left(R_{\nu} e^{-\sigma(X-D)}+S_{\nu} e^{\sigma(X-D)}\right) d \nu
\end{aligned}
$$

where $\sigma^{2}=\nu^{2}+\rho^{2}\left(\beta^{2}-k^{2} n_{2}^{2}\right)=\nu^{2}+W^{2}$. Finally, we relate the cylindrical forms of the cladding fields (2) to these Cartesian forms (7), and we equate the slab fields at the cladding-PWG boundary to the fields of (7) to complete the set of equations. The former problem has been addressed in detail in [1], [2], and is reproduced for the present geometry in 


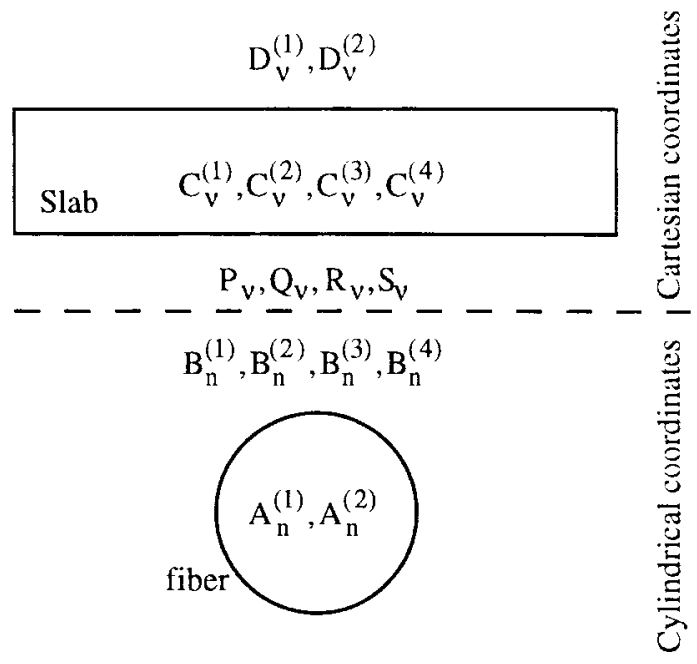

Fig. 2. The field coefficients in the various areas. Note that two different representations of the cladding fields are used: in Cartesian and cylindrical coordinates.

Appendix A. The latter problem is a straight-forward boundary value problem.

At this point we have expressed the coefficients in the various expansions as linear combinations of coefficients of other expansions, forming a set of linear equations. In Fig. 2 we provide a diagram showing the field coefficients and their respective regions.

Upon substitution of all boundary constraints [(1)-(4) and (7)] into (5) and (6), we arrive at a system of $2 n$ equations of $2 n$ unknown coefficients (e.g., $A_{n}^{(1)}$ and $A_{n}^{(2)}$ ) and propagation constant $\beta$, which may be expressed in matrix form as

$$
\left[\begin{array}{ll}
K 1_{m, n} & K 2_{m, n} \\
K 3_{m, n} & K 4_{m, n}
\end{array}\right]\left[\begin{array}{l}
A_{n}^{(1)} \\
A_{n}^{(2)}
\end{array}\right]=[0] .
$$

Here, $K 1, K 2, K 3, K 4$ are submatrices with elements that are complicated functions of integral equations. These relations also are found in Appendix B. This complex system of equations has nontrivial solutions when its determinant is zero. As we may use the propagation constant $\beta$ as a variable in these relations, the roots of the equation provide mode solutions for the composite waveguide structure. Having found the roots, we next calculate the field coefficients by back substitution of our solution into (8).

The system of equations generated by the mathematical model described in Fig. 2 has been solved using standard mathematical libraries for Bessel function evaluation, matrix evaluation and numerical integration. The modes of the coupler have been identified by calculating the determinant of the matrix of (8), using four $20 \times 20$ submatrices, $K 1, K 2$, $K 3$, and $K 4$. Then, the expansion coefficients for the various modes have been calculated by back-substituting the solution of $\beta$ into (8). The results obtained for different configurations are presented in the next section. The computation time required to calculate a determinant root of the matrix in (8) varies with the integral accuracy. When we use 40 point abcissas and weights in the Gaussian quadrature calculation, the computation time is less than $7 \mathrm{~s}$ with a $166 \mathrm{MHz}$ Pentium machine.

\section{MODAl SOLUTIONS OF THE COUPLER}

The roots of the determinant of the coefficient matrix have been calculated numerically, with the modal propagation constant $\beta$ determining the mode solution. While several mode solutions are identified for any given geometry and accuracy of solution, we classify these solutions into two groups, namely, ridge modes and unbounded continuous modes. The ridge modes are well confined in the region of the slab-fiber system and are very stable with the change of computation accuracy, namely, numerical integration and the number of components in the field expansion.

The unbounded modes are distinguished by lack of boundedness in the $y$-direction and lack of solution stability with increasing accuracy of numerical calculation. The stability issue might lead one to believe that these are spurious solutions; however, the observation that these modes consistently satisfy the boundary conditions over the regions of interest lead us to conclude that they are solutions within the continuum of unbounded slab modes.

In Fig. 3 we show the characteristics of the $q$-TM mode solutions of the evanescent coupler with changing fiber core index $n_{1}$ using the following parameters: $n_{2}=1.46, n_{3}=$ $1.4745, n_{4}=1.46, \lambda=1.3 \mu \mathrm{m}, \rho=2.5 \mu \mathrm{m}, s=3 \mu \mathrm{m}$. The upper limit in the propagation constant $\left(k n_{3}\right)$ and the lower limit $\left(k n_{2}\right)$ are due to the slab and the cover medium indexes, respectively, which must bound any modes guided by the slab structure. The solution [10] for an isolated single-mode fiber with the corresponding values of $n_{1}, n_{2}$, and $\rho$ has been superimposed on the curves of Fig. 3 for reference, and is denoted "fiber mode." The unbounded modes have continuous propagation constants in the region labeled the unbounded mode region. In this region, we identify even modes, which have the same field direction in the slab and the fiber and are located above the isolated fiber curve. The odd unbounded modes occupy the region below the isolated fiber solution; these modes are characterized by a zero crossing between the field lobes in the slab and the fiber core region.

The ridge mode, which has the largest propagation constant, is the only mode that is well-bounded in the vicinity of the fiber core (even when the field is primarily in the slab). For this mode, we plot the modal fields (Fig. 4) at the solution points A, B, C, and D of Fig. 3. The ridge mode approaches the fiber mode asymptotically with increasing $n_{1}$, particularly as $n_{1}$ exceeds the slab index, eventually becoming a perturbed fiber mode. Fig. 4 also shows the transfer of power between the two waveguides, as the core refractive index increases. Near point A, the fiber core acts as a weak attractor for the slab fields, providing only weak guidance for the slab mode. However, near point $\mathrm{D}$, the field is nearly a fiber mode solution, with tight field confinement in the slab near the core region.

The comparison of $q$-TE and $q$-TM modes, shown in Table I, reveals that the differences in propagation constants, $\beta$, for these configurations are very small as stated in [8]. Therefore, we have considered only $q$-TM solutions throughout this analysis; however we provided the expressions for $q$-TE modes in Appendix C. 


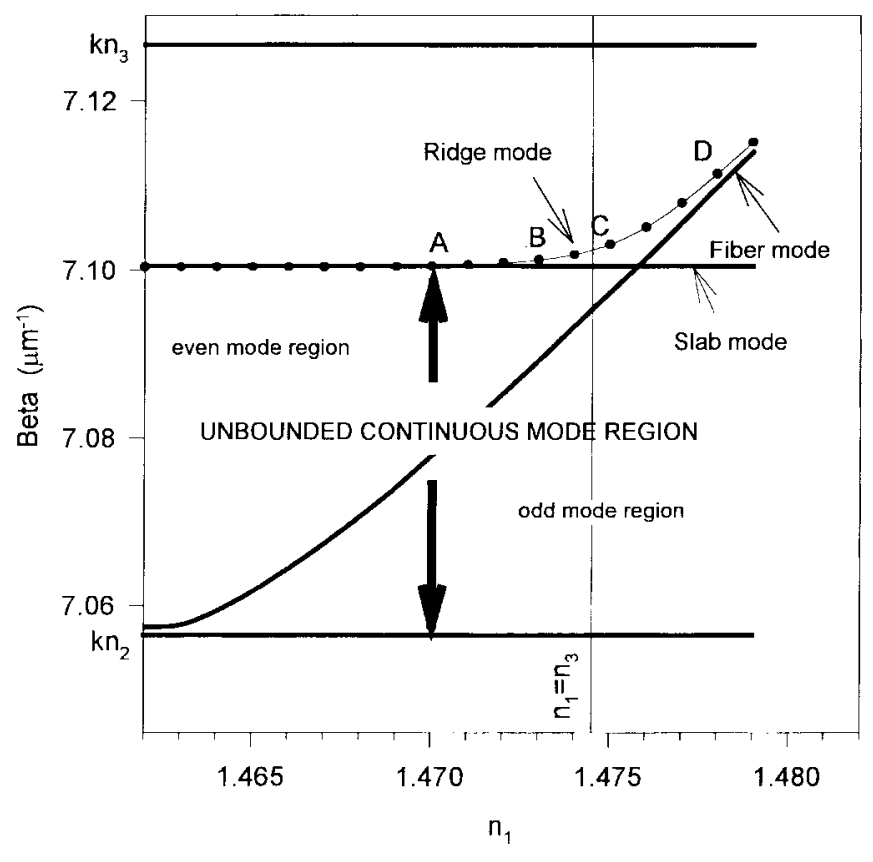

Fig. 3. Mode propagation constants of the evanescent coupler versus $n_{1}$ for $n_{3}=1.4745, n_{2}=n_{4}=1.46, \rho=2.5 \mu \mathrm{m}, s=3 \mu \mathrm{m}$, and $d=3 \mu \mathrm{m}$. The region labeled the leaky mode region contains a continuum of solutions.

We compare our results with propagation constants of the ridge modes obtained using CMT [2] for the same configuration in Table I. Although only small differences are observed in $\beta$, the differences in the transverse propagation constants, which dictate mode profiles and, hence, coupling coefficients, may be quite large. In the examples shown, the difference in transverse propagation constant is found to be as large as $13 \%$. The maximum error occurs when the fiber core index is much lower than the slab index, where the effect of the slab perturbs the fiber solution strongly.

In Fig. 3, a gap is observed between the ridge mode solution and the upper limit of the unbounded mode region. This gap may be explained by considering the modal parameters of the planar waveguide. The relationship between the propagation constants in the rectangular coordinate system is

$$
k^{2} n_{3}^{2}-\tau^{2} / \rho^{2}=\beta^{2}+\nu^{2} / \rho^{2} .
$$

In this equation, the left-hand side is nearly constant for a mode of the planar waveguide because $\tau$, the propagation constant in the $x$-direction, must provide for approximately one-half wavelength across the slab (for lower-order modes). The right-hand side of the equation includes $\nu$, the propagation constant in the $y$-direction, which must be complex for a bounded mode. When $\nu$ becomes complex for the bounded mode, the propagation constant $\beta$ must increase to maintain equality, and thus a bounded ridge mode must have a larger propagation constant than an unbounded slab mode. In the limit of small $n_{1}$, we observe that the ridge mode solution asymptotically approaches the unbounded slab mode value.

\section{POWER TRANSMISSION}

We now use these observations to consider the optical power transmission behavior of a tunable device, where the overlay
TABLE I

Comparison of Propagation Constant Solutions BetweEn CMT AND MODE EXPANSION THEORY

\begin{tabular}{l|l|l|l}
\hline $\mathrm{n}_{1}$ & $\beta(\mathrm{CMT})$ & $\beta_{\text {ridge }}$ (TE) & $\beta_{\text {ridge }}(\mathrm{TM})$ \\
\hline 1.4817 & 7.125755 & 7.1258700 & 7.1258701 \\
\hline 1.4756 & 7.104438 & 7.1043102 & 7.1042100 \\
\hline 1.4745 & 7.103230 & 7.1025700 & 7.1023902 \\
\hline 1.4709 & 7.102521 & 7.1009098 & 7.1006301 \\
\hline
\end{tabular}

index may be adjusted (e.g., electro-optically). The device geometry is pictured in Fig. 5, where we assume that singlemode fibers are coupled to a device of length $L_{c}$ at the input and output. The fiber parameters are identical to the "fiber" of the coupler.

We note that unbounded modes in the $y$-direction will not effectively transfer power through the coupler; instead, any power coupled into these modes will be transferred outward from the core region so that very little power may be coupled to an output fiber after propagation through the device. We also observe that the best power transmission, i.e., strong coupling between the device and input/output fibers, will occur in cases where the ridge mode behaves as a perturbed fiber mode, such as in Fig. 4(d). Here, the field overlap will be strong for launching the ridge mode and for coupling the ridge mode to the output fiber.

We begin by considering the case where $n_{3}$ is large and variable, so that more than one ridge mode solution is allowed in the planar waveguide. Fig. 6 shows the dispersion curve for the two ridge modes (unbounded modes are omitted here), with parameters $n_{1}=1.4646, n_{2}=n_{4}=1.46, \lambda=$ $0.85 \mu \mathrm{m}, \rho=2.5 \mu \mathrm{m}$, and $s=3 \mu \mathrm{m}$. The horizontal line at the bottom represents the behavior of the isolated fiber mode solution (which is independent of $n_{3}$ ). The top horizontal line corresponds to the plane wave propagation constant of the core $\left(k n_{1}\right)$. When the slab index $\left(n_{3}\right)$ approaches the cladding index $\left(n_{2}\right)$, the solution for $\beta$ of the first ridge mode approaches that of the isolated fiber, as expected. As $n_{3}$ increases, the first ridge mode deviates from the isolated fiber solution, approaching $k n_{1}$. Beyond this value, the first ridge mode is no longer strongly guided by the core, becoming an unbounded slab mode (i.e., fields extend indefinitely in the $y$-direction). Here, the mode is essentially a weakly perturbed slab mode carrying very little power in the core region.

As $n_{3}$ increases further, a second, odd, ridge mode solution is produced with an initial propagation constant very nearly that of the isolated fiber. Thus, just beyond the cutoff for this mode, most of its power is located in the core region of the fiber, as in Fig. 4(d). Further increase of $n_{3}$ leads to weaker guidance in the core region until the mode becomes unbounded; this occurs more rapidly for the second ridge mode due to its higher spatial frequency in the $x$-direction.

Transmission of optical power through the coupler may be calculated by means of coupling to ridge modes. The power carried by the input optical fiber is first decomposed into the ridge modes, propagated through the fiber/slab structure, and launched into the second fiber. We neglect any power coupled 


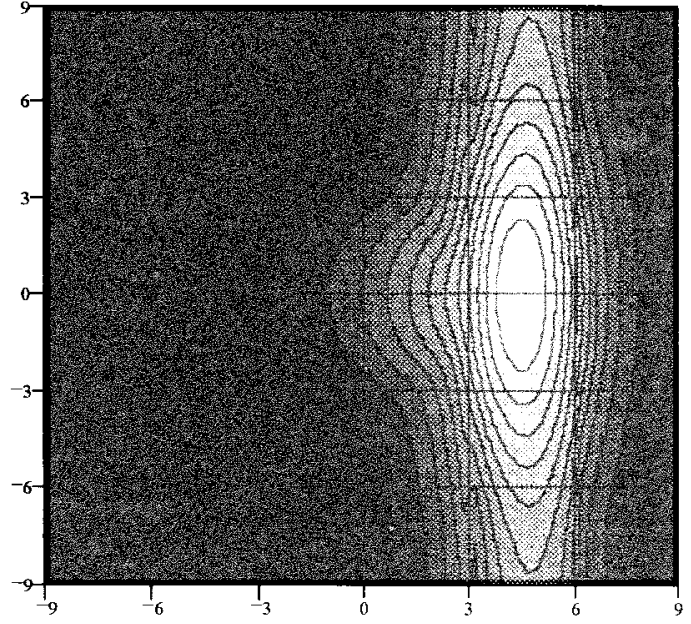

(a)

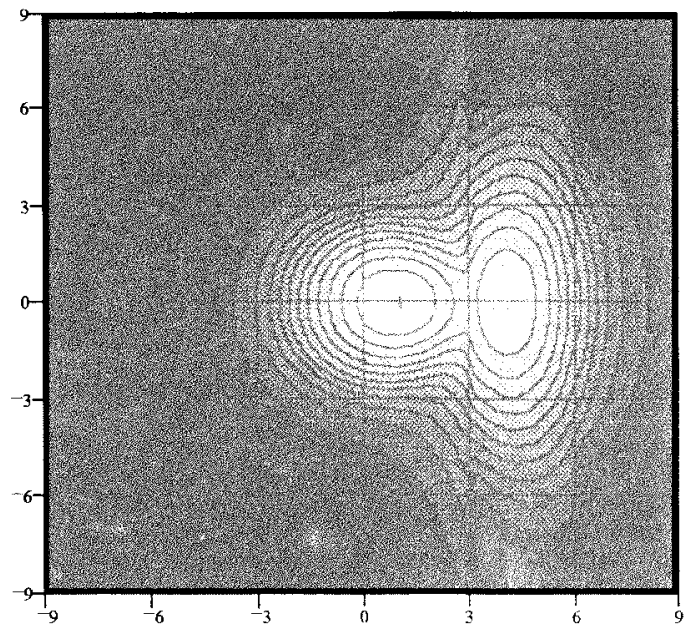

(c)

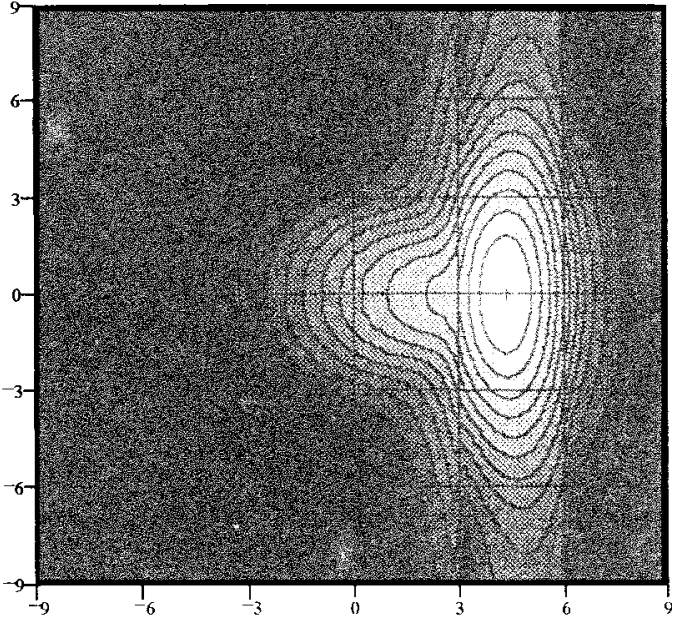

(b)

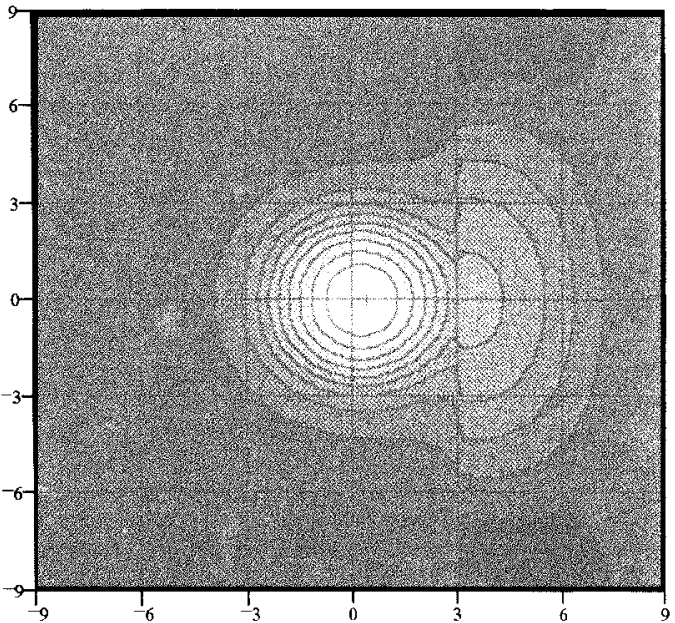

(d)

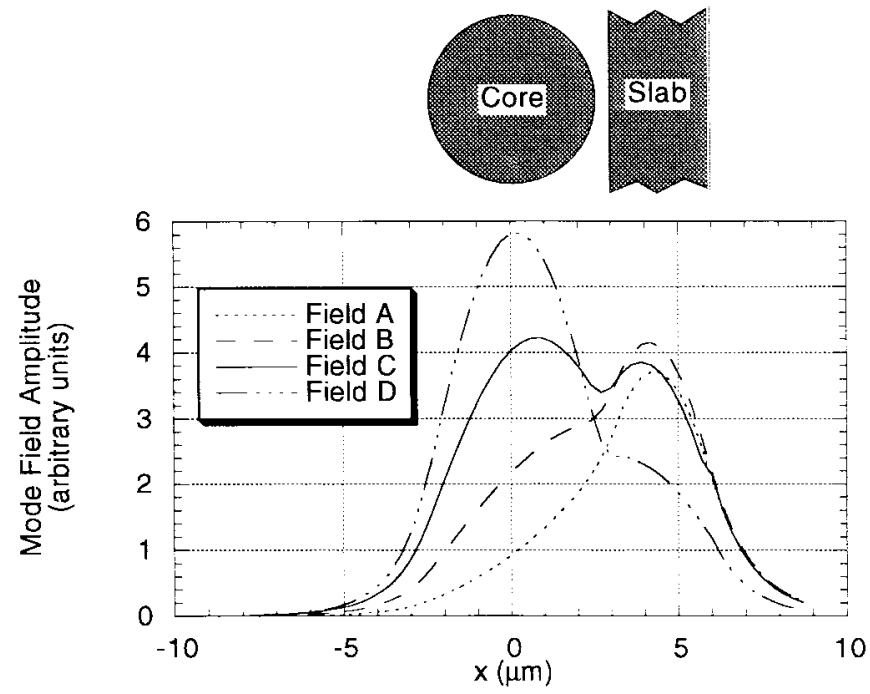

(e)

Fig. 4. The transverse E-fields of the ridge mode at the points A, B, C, and D shown in Fig. 3. (a) $n_{1}=1.47$, (b) $n_{1}=1.473$, (c) $n_{1}=1.475$, (d) $n_{1}=1.478$, and (e) the field magnitudes on the $x$-axis for the cases A, B, C, and D.

into unbounded modes in this analysis, assuming such power to be completely dissipated at the device output. Because the first ridge mode becomes unbounded prior to the onset of the second ridge mode, we may consider the modes individually over their respective regions of $n_{3}$ where significant coupling occurs. 


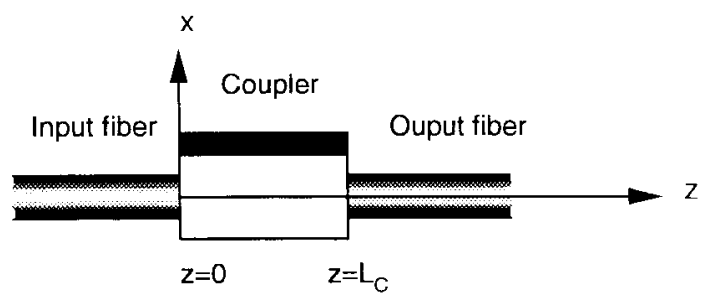

Fig. 5. Model for power transmission calculations for the evanescent coupler with fiber pigtails.

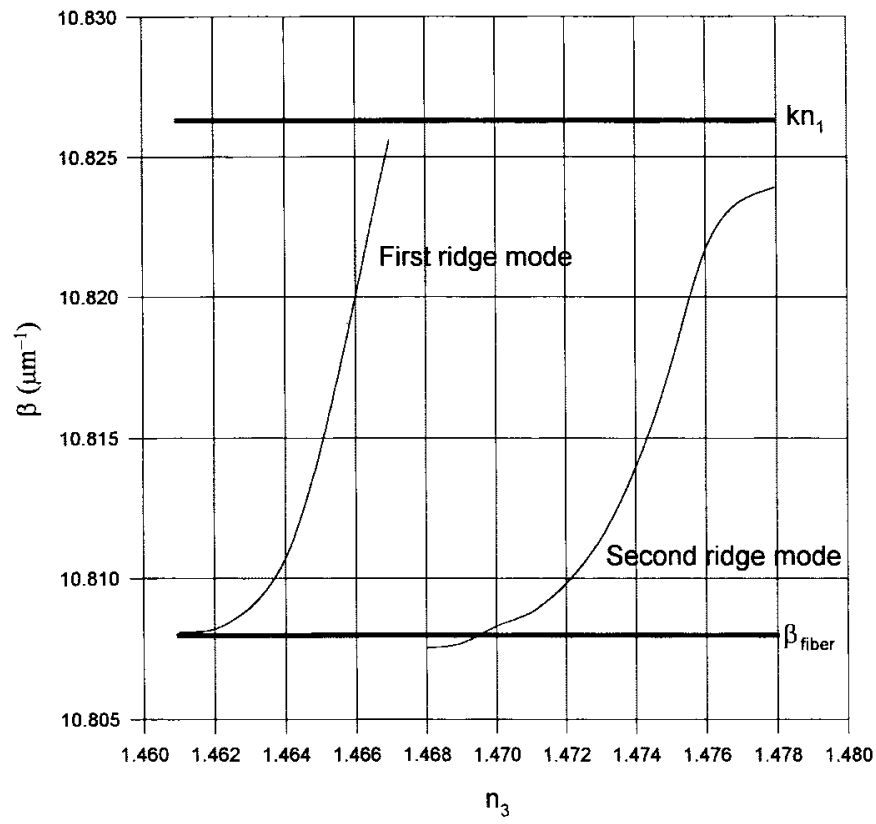

Fig. 6. Propagation constant solutions for the ridge modes versus $n_{3}$ for $n_{1}=1.4646, n_{2}=n_{4}=1.46, \rho=2.5 \mu \mathrm{m}, s=3 \mu \mathrm{m}$, and $d=3 \mu \mathrm{m}$.

In Fig. 7, we plot the transmission versus $n_{3}$ for our model, and for the geometry described above. We observe that the power transmission peaks when the ridge mode field is concentrated in the fiber core region, corresponding to maximum overlap between the waveguide modes. As $n_{3}$ is increased, the ridge mode field becomes concentrated in the slab region, and the overlap between modes smoothly approaches zero. As $n_{3}$ increases further, higher-order ridge modes are launched, producing efficient power transmission over ranges where the particular ridge mode field approximates the fiber mode field.

\section{Dependence on Guide Separation: CONVERGENCE TO IsOlated Guide MODES}

We next investigate the dependence of the ridge mode solutions on the separation between the fiber core and the slab waveguide. We consider two cases: i) when $n_{1}<n_{3}$ and ii) when $n_{1}>n_{3}$. As we shall see, the ridge mode solution will converge to the isolated waveguide solution of the strongest guide as the separation increases, so the two cases must be considered separately.

First, consider the case when $n_{3}>n_{1}$, so that the slab index is greater than the core index. The propagation constant for

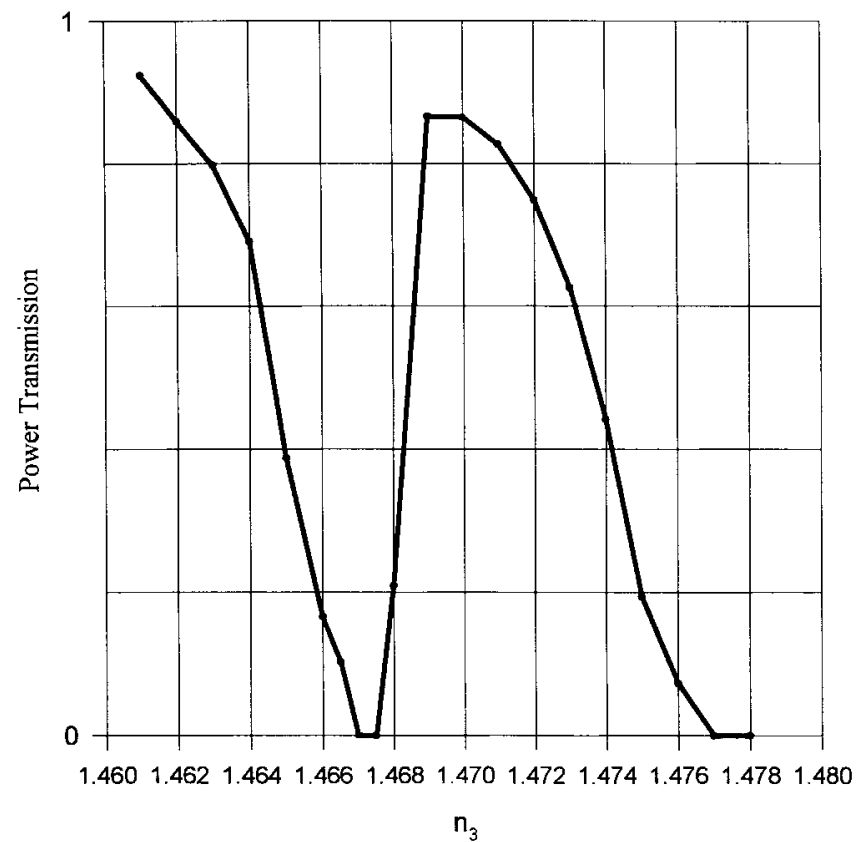

Fig. 7. Power transmission characteristic of the coupler versus $n_{3}$.

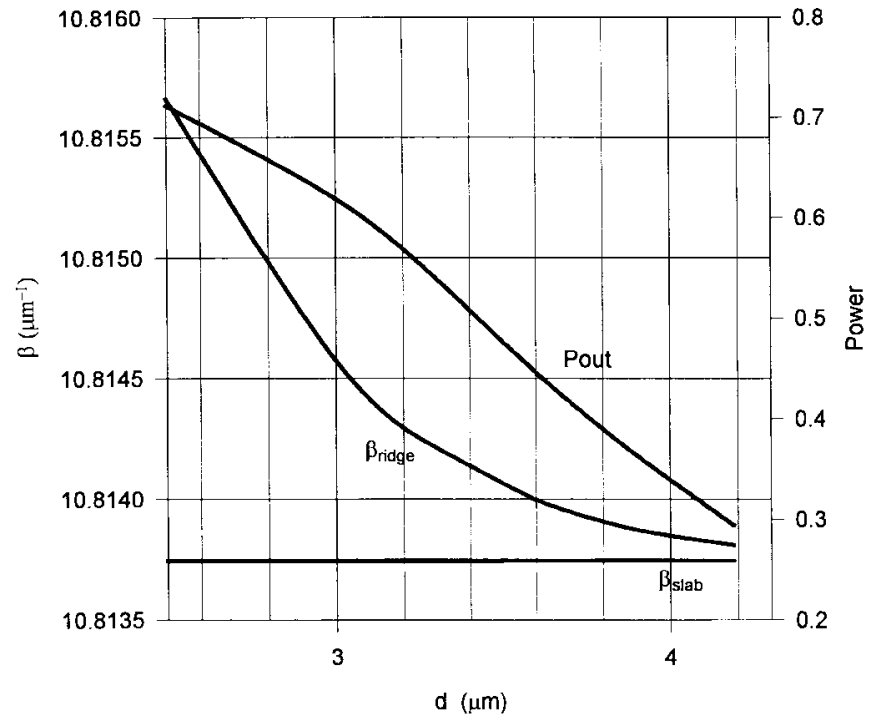

Fig. 8. Propagation constant solution versus guide separation $\left(n_{3}>n_{1}\right)$. Note the convergence of the ridge mode to the slab mode.

the ridge mode and the transmitted power are plotted versus guide separation $d$ in Fig. 8 for the following parameters: $n_{1}=$ $1.4646, n_{3}=1.465, n_{2}=n_{4}=1.46, \rho=2.5 \mu \mathrm{m}, s=3 \mu \mathrm{m}$, and $\lambda=1.3 \mu \mathrm{m}$. We observe that the ridge mode solution converges to the isolated slab propagation constant, $\beta=$ 10.813745 , as the separation increases, converging beyond $d \approx 4.2 \mu \mathrm{m}$. Here, the power transmission through the device is reduced toward zero due to two effects. In addition to the increasing guide separation, which tends to pull the field lobe toward the slab guide, the ridge mode solution extends further in the $y$-direction as the separation $\mathrm{d}$ increases, eventually converging to an unbounded slab mode as the interaction between the slab and the fiber core becomes negligible. 


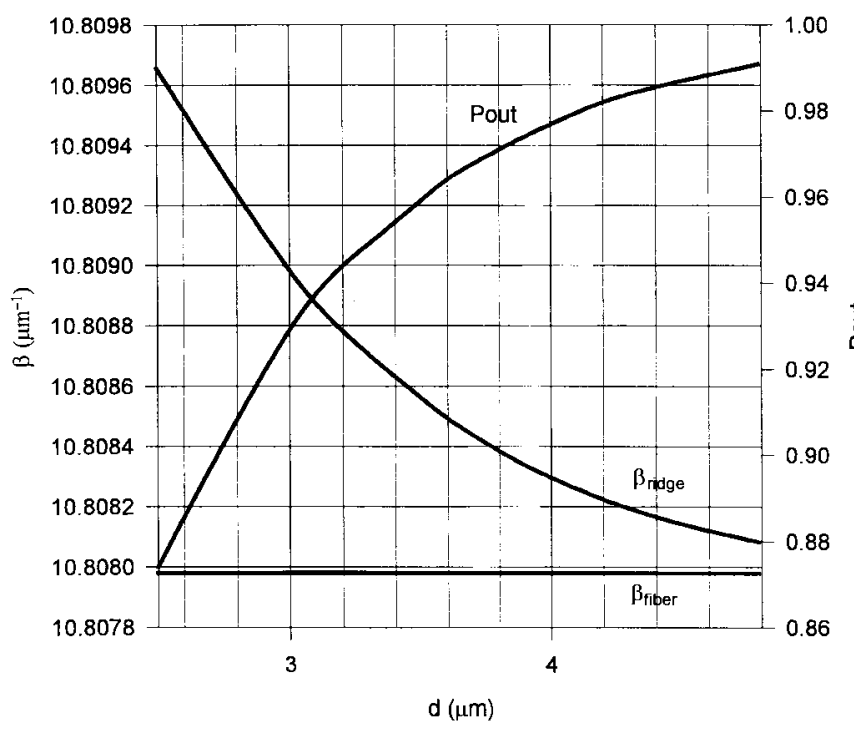

Fig. 9. Propagation constant solution versus guide separation $\left(n_{1}>n_{3}\right)$. Note the convergence of the ridge mode to the fiber mode.

Now consider the case when $n_{1}>n_{3}$, where the fiber core index exceeds the slab index. We take as an example the same parameters as in the preceding case, except that the slab index is reduced to 1.463. Fig. 9 shows the dependence of the ridge mode propagation constant and the transmitted power on the guide separation in this case. Here, the solution converges to the isolated fiber solution, with $\beta$ approaching 10.808 as the separation increases. Again, beyond a separation of about $4.2 \mu \mathrm{m}$, the ridge mode behaves as the fundamental mode of the fiber. This is verified by the large power transmission through the device as the separation is increased, indicating only a slight perturbation in the fiber mode.

It is therefore clear that our solution for the ridge mode behaves asymptotically as expected, converging to the isolated waveguide solution for one of the two interacting guides. However, we observe that the solution always converges to the fundamental mode solution of the strongest waveguide, although in reality both isolated guides should propagate power independently. When the convergent mode is the slabguided mode, large guide separation causes the solution to depend strongly on the accuracy of the computation so that the ridge mode becomes indistinguishable from the unbounded continuous slab modes.

\section{CONCLUSION}

We have investigated the vector mode solutions of the evanescent coupler comprising a slab and a single mode optical fiber. The method which we have used in this paper has been developed by Marcuse [8] for analyzing a fiber system having a cladding limited by an infinite half-space. We have expanded this analysis to the fiber/slab coupler system, formulating the full coefficient matrix of the mode expansions and incorporating corrections to minor errors in the original derivation of [8].
By solving the coefficient matrix as explained in the text, we have found ridge modes and unbounded modes. However, when these models are scrutinized, it is apparent that only one ridge mode, if any, is well bounded at any one time. This explains why modal interference effects predicted by CMT have been not observed. This fundamental result makes this coupler different than the other couplers which rely on the beating of those two modes, namely, odd and even ridge modes.

The propagation constant solutions of the CMT and the vector modal method have been compared for a variety of configurations. We have found that CMT produces errors in the computation of transverse propagation constants, particularly in the case of large slab index or close waveguide proximity, and thus will produce inaccurate predictions for optical transmission in many geometries.

In addition, we have investigated the effect of separation distances between the two waveguides on the propagation constant $\beta$ and on transmitted power. We found that increasing the separation distance causes the ridge mode to converge to the individual waveguide mode, either a slab or a fiber mode depending on the initial configuration of the coupler. We also note that our analysis remains valid even in the case of close proximity $(d \rightarrow 0)$, where the assumptions of CMT break down.

The optical fiber used in these calculations has been assumed to support the fundamental mode only, but the model is applicable to higher order mode solutions as well. Also an asymmetric PWG can be used in the model by choosing the cladding index $\left(n_{2}\right)$ and cover medium index $\left(n_{3}\right)$ to be different. Finally, although the spectral response of the device has not been analyzed here, application of this model to the design of wavelength filters is straightforward, and shall be addressed in a future publication.

\section{APPENDIX}

These appendices are provided to serve two purposes. First, we provide mathematical relationships between expansion coefficients in our mathematical model and give the explicit forms of the $K 1, K 2, K 3$, and $K 4$ submatrices. Second, we provide corrections to typographic errors that we have identified in [8], the foundation of this formulation.

The relevant errors in [8] that affect the analysis consist of sign errors and transposition of matrix indexes. In Appendix B, we provide the applicable expressions, which are quite similar to those of [8], and the equation sign convention and index order provided here may be applied to the corresponding equations in [8] in order to correct the latter. One exception is (30) of [8], for a parallel is not provided here; the correct equation should read

$$
\kappa=\left(\sigma Q_{3}^{2}+\tau Q_{2}^{2}\right)\left(n_{2}^{2} \sigma Q_{3}^{2}+n_{3}^{2} \tau Q_{2}^{2}\right)-\nu^{2} n_{n \text { eff }}^{2} V_{3}^{4} .
$$

\section{APPENDIX A}

The following relations between the cladding field expansion coefficients in the Cartesian and cylindrical representations have been derived by Marcuse [8] and Vassallo [9]. We 
provide them here for completeness

$$
\begin{aligned}
B_{n}^{(2)} & =\frac{2}{e_{n}} \int_{-\infty}^{\infty} Q_{\nu} \exp (-\sigma D) \sigma \cosh (n g) d g \\
B_{n}^{(4)} & =\frac{2 j}{e_{n}} \int_{-\infty}^{\infty} S_{\nu} \exp (-\sigma D) \sigma \sinh (n g) d g \\
P_{\nu} & =\frac{\exp (-\sigma D)}{2 \sigma} \sum_{n=0}^{\infty} B_{n}^{(1)} \cosh (n g) \\
R_{\nu} & =\frac{-j \exp (-\sigma D)}{2 \sigma} \sum_{n=0}^{\infty} B_{n}^{(3)} \sinh (n g)
\end{aligned}
$$

where

$$
e_{n}=\left\{\begin{array}{cc}
2 & n=0 \\
1 & \text { otherwise }
\end{array}\right\} \text { and } g=\cosh ^{-1}\left(\frac{\sigma}{W}\right)
$$

\section{APPENDIX B}

As stated in the text, applications of the boundary conditions at the various interfaces and the equating of the cladding field representations provided in Appendix A allow the construction of a coefficient matrix. Upon substitution of these conditions into two of the boundary equations [we use (5) and (6) for convenience], the cladding coefficients may be expressed in a form

$$
\begin{aligned}
Q_{\nu} & =\hat{A} P_{\nu}+j \hat{B} R_{\nu} \\
S_{\nu} & =j \hat{C} P_{\nu}+\hat{D} R_{\nu} .
\end{aligned}
$$

Here, $\hat{A}, \hat{B}, \hat{C}$, and $\hat{D}$ may be regarded as reflection coefficients relating the exponentially increasing and decreasing components of the cladding fields. Although these terms may be expressed in closed form, the expressions are quite cumbersome and best solved in parts. We will not provide their forms here, although their calculation is quite straightforward.

The terms $P_{\nu}$ and $R_{\nu}$ are expressed in (A.4) and (A.5) in terms of $B_{n}^{(1)}$ and $B_{n}^{(3)}$, respectively. By substituting these equations into (B.1) and (B.2), we obtain $Q_{\nu}$ and $S_{\nu}$ in terms of $B_{n}^{(i)}$. The (A.2) and (A.3) can be expressed in four coefficients $B_{n}^{(i)}$ upon substitution of the $Q_{\nu}$ and $S_{\nu}$. The last step to complete the eigensystem is to use the relations between the coefficients of cladding and core obtained at the core/cladding boundary. Then these two equations, in terms of $A_{n}^{(1)}$ and $A_{n}^{(2)}$, can be arranged in the matrix form of (8). Here the submatrix expressions have the following forms:

$$
\begin{aligned}
K 1_{m, n}= & \frac{n}{e_{m}} \frac{n_{\mathrm{eff}}}{Z_{0}} \frac{V^{2}}{U^{2}} J_{n}(U) I_{n}(W) B_{n, m}+A_{n, m} \frac{1}{e_{m}} \frac{W}{U} \\
& \cdot\left(W \frac{n_{1}^{2}}{n_{2}^{2}} J_{n}^{\prime}(U) I_{n}(W)+U I_{n}^{\prime}(W) J_{n}(U)\right) \\
+ & \delta_{n, m} \frac{W}{U}\left(W \frac{n_{1}^{2}}{n_{2}^{2}} J_{n}^{\prime}(U) K_{n}(W)+U\right. \\
K 2_{m, n}= & n \frac{n_{\mathrm{eff}}}{n_{2}^{2}} \frac{V^{2}}{U^{2}} Z_{0} J_{n}(U) K_{n}(W) \delta_{n, m}+A_{n, m} \frac{n}{e_{m}} \frac{n_{\mathrm{eff}}}{n_{2}^{2}} \\
& \cdot \frac{V^{2}}{U^{2}} Z_{0} J_{n}(U) I_{n}(W)+B_{n, m} \frac{W}{U} \frac{1}{e_{m}} \\
& \cdot\left(W J_{n}^{\prime}(U) I_{n}(W)+U I_{n}^{\prime}(W) J_{n}(U)\right)
\end{aligned}
$$

$$
\begin{aligned}
K 3_{m, n}= & n \frac{n_{\mathrm{eff}}}{Z_{0}} \frac{V^{2}}{U^{2}} J_{n}(U) K_{n}(W) \delta_{n, m}+D_{n, m} \frac{1}{e_{m}} \frac{n_{\mathrm{eff}}}{Z_{0}} \\
& \cdot \frac{V^{2}}{U^{2}} J_{n}(U) I_{n}(W)-C_{n, m} \frac{1}{e_{m}} \frac{W}{U} \\
& \cdot\left(W \frac{n_{1}^{2}}{n_{2}^{2}} J_{n}^{\prime}(U) I_{n}(W)+U I_{n}^{\prime}(W) J_{n}(U)\right) \\
K 4_{m, n}= & \frac{-n}{e_{m}} \frac{n_{\mathrm{eff}}}{n_{2}^{2}} \frac{V^{2}}{U^{2}} Z_{0} J_{n}(U) I_{n}(W) C_{n, m}+D_{n, m} \frac{1}{e_{m}} \\
& \cdot \frac{W}{U}\left(W J_{n}^{\prime}(U) I_{n}(W)+U \quad I_{n}^{\prime}(W) J_{n}(U)\right) \\
& +\delta_{n, m} \frac{W}{U}\left(W J_{n}^{\prime}(U) K_{n}(W)+U K_{n}^{\prime}(W)\right. \\
& \left.\cdot J_{n}(U)\right)
\end{aligned}
$$

where $n_{\mathrm{eff}}=\beta / k$.

We emphasize that these equations are similar in form to those of [8], with sign errors corrected in (B.4) and (B.5). Also, the order of the indexes of the $A_{n, m}, B_{n, m}, C_{n, m}$, and $D_{n, m}$ matrix elements differ from those of the reference, and an additional term (the ratio of the refractive indexes) has been incorporated into (B.3).

The matrix elements may be found from the reflection coefficients defined in (B.1) and (B.2) using

$$
\begin{aligned}
& A_{n, m}=\int_{-g_{m}}^{g_{m}} \hat{A} \exp (-2 \sigma D) \cosh (n g) \cosh (m g) d g \\
& B_{n, m}=\int_{-g_{m}}^{g_{m}} \hat{B} \exp (-2 \sigma D) \sinh (n g) \cosh (m g) d g \\
& C_{n, m}=\int_{-g_{m}}^{g_{m}} \hat{C} \exp (-2 \sigma D) \cosh (n g) \sinh (m g) d g \\
& D_{n, m}=\int_{-g_{m}}^{g_{m}} \hat{D} \exp (-2 \sigma D) \sinh (n g) \sinh (m g) d g .
\end{aligned}
$$

The limits of integration are constrained by the maximum allowed spatial frequency $\left(\nu_{\max }=W \sinh \left(g_{m}\right)\right)$. For a given eigenvalue $\beta$, the value is constrained by $\nu^{2} \leq \nu_{\max }^{2}=$ $\left(k^{2} n_{3}^{2}-\beta^{2}\right) r h o^{2}$.

\section{APPENDIX C}

The relations below are derived for $q$-TE modes. The cladding fields are related by the following equations:

$$
\begin{aligned}
B_{n}^{(2)} & =\frac{j 2}{e_{n}} \int_{-\infty}^{\infty} Q_{v} \exp (-\sigma D) \sigma \sinh (n g) d g \\
B_{n}^{(4)} & =\frac{2}{e_{n}} \int_{-\infty}^{\infty} S_{v} \exp (-\sigma D) \sigma \cosh (n g) d g \\
P_{v} & =-j \frac{\exp (-\sigma D)}{2 \sigma} \sum_{n=0}^{\infty} B_{n}^{(1)} \sinh (n g) \\
R_{v} & =\frac{\exp (-\sigma D)}{2 \sigma} \sum_{n=0}^{\infty} B_{n}^{(3)} \cosh (n g)
\end{aligned}
$$

where $e_{n}$ is same for both polarizations. 
The submatrices $K 1, K 2, K 3$, and $K 4$ assume the following integral expressions $A_{n, m}, B_{n, m}, C_{n, m}$, and $D_{n, m}$ for $q$-TE modes

$$
\begin{aligned}
& A_{n, m}=\int_{-g_{m}}^{g_{m}} \hat{A} \exp (-2 \sigma D) \sinh (n g) \sinh (m g) d g \\
& B_{n, m}=\int_{-g_{m}}^{g_{m}} \hat{B} \exp (-2 \sigma D) \sinh (m g) \cos (n g) d g \\
& C_{n, m}=\int_{-g_{m}}^{g_{m}} \hat{C} \exp (-2 \sigma D) \cosh (m g) \sinh (n g) d g \\
& D_{n, m}=\int_{-g_{m}}^{g_{m}} \hat{D} \exp (-2 \sigma D) \cosh (n g) \cosh (m g) d g .
\end{aligned}
$$

Then (8) for $q$-TE modes becomes

$$
\left[\begin{array}{rr}
K 1_{m, n} & -K 2_{m, n} \\
-K 3_{m, n} & K 4_{m, n}
\end{array}\right]\left[\begin{array}{l}
A_{n}^{(1)} \\
A_{n}^{(2)}
\end{array}\right]=[0]
$$

where the matrix elements are found by substituting (C.5)-(C.8) into (B.3)-(B.6).

\section{REFERENCES}

[1] D. Marcuse, "Investigation of coupling between a fiber and infinite slab," J. Lightwave Technol., vol. 7, pp. 122-130, Jan. 1989.

[2] S. Zheng, Lee N. Binh, and G. P. Simon, "Light coupling and propagation in composite optical fiber-slab waveguide," J. Lightwave Technol., vol. 13, pp. 244-251, Feb. 1995.

[3] P. P. Krassimir and A. T. Andreev, "Distributed coupling between a single-mode fiber and a planar waveguide," J. Opt. Soc. Amer. B, vol. 11, pp. 826-834, May 1994
[4] W. Johnstone, G. Thursby, D. Moodie, R. Varshrey, and B. Culshaw, "Fiber optic wavelength channel selector with high resolution," Electron. Lett., vol. 28, pp. 1364-1366, July 1992.

[5] K. McCallion and W. Johnstone, "Tunable in-line fiber-optic bandpass filter," Opt. Lett., vol. 19, pp. 542-544, Apr. 1993.

[6] G. Fawcett, W. Johnstone, I. Andonovic, D. J. Bone, T. G. Harvey, N. Carter, and T. G. Ryan, "In line fiber-optic intensity modulator using electro-optic polymer," Electron. Lett., vol. 28, pp. 984-986, May 1992.

[7] K. McCallion, W. Johnstone, and G. Thursby, "Investigation of optical fiber switch using electro-optic interlays," Electron. Lett., vol. 28, pp. 409-411, Feb. 1992.

[8] D. Marcuse, F. Ladouceur, and J. D. Love, "Vector modes of $D$-shaped fibers," Inst. Elec. Eng. Proc. J., vol. 139, pp. 117-126, Apr. 1992.

[9] C. Vassallo, "Rigorous theory for modes of optical fibers with cladding limited by a plane," Electron. Lett., vol. 22, pp. 944-946, Aug. 1986.

[10] Allan W. Snyder and John D. Love, Optical Waveguide Theory. New York: Chapman \& Hall 1991, ch. 14.

Mehmet S. Dinleyici received the B.S. and M.Sc. degrees in electrical engineering from University of Gaziantep, Gaziantep, Turkey, in 1991 and 1993, respectively. He is currently working towards the Ph.D. degree at the Illinois Institute of Technology, Chicago.

He is recipient of a scholarship provided by Izmir Institute of Technology, Izmir, Turkey. His interest is in the area of optoelectronic, fiber optics, optical fiber devices, and dielectric waveguides.

David B. Patterson (M'93) was born in Greenfield, IA, on June 13, 1962 He received the B.S. degree in physics from Iowa State University, Ames, in 1984, and the M.S. and Ph.D. degrees in applied physics from Stanford University, Stanford, CA, in 1988 and 1990, respectively.

Following his doctoral work on acoustooptic fiber modulators, he was employed as a Research Associate at Stanford University, working on the development of in-fiber frequency shifters. He joined the Electrical and Computer Engineering Department at the Illinois Institute of Technology in 1991 and was promoted to Associate Professor in 1997. His current research interests include acoustooptic fiber modulators, grating-based dispersion compensation, quantum cryptography, and optical waveguide components for photonic applications. 